\title{
Stepwise photosensitized thymine dimerization mediated by an exciton intermediate
}

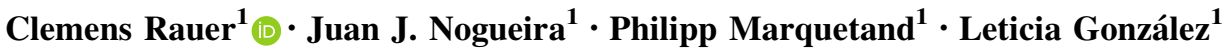

Received: 16 October 2017/Accepted: 20 November 2017/Published online: 4 December 2017

(c) The Author(s) 2017. This article is an open access publication

\begin{abstract}
Cyclobutane thymine dimerization is the most prominent DNA photoinduced damage. While the ultrafast mechanism that proceeds in the singlet manifold is nowadays well established, the triplet-state pathway is not completely understood. Here we report the underlying mechanism of the photosensitized dimerization process in the triplet state. Quantum chemical calculations, combined with wavefunction analysis, and nonadiabatic molecular dynamics simulations demonstrate that this is a stepwise reaction, traversing a long-lived triplet biradical intermediate, which is characterized as a Frenkel exciton with very small charge-transfer character. The low yield of the reaction is regulated by two factors: (i) a relatively large energy barrier that needs to be overcome to form the exciton intermediate, and (ii) a bifurcation of the groundstate potential-energy surface that mostly leads back to the Franck-Condon region because dimerization requires a very restricted combination of coordinates and velocities at the event of non-radiative decay to the ground state.
\end{abstract}

Clemens Rauer

clemens.rauer@univie.ac.at

1 Institute of Theoretical Chemistry, Faculty of Chemistry, University of Vienna, Vienna, Austria

\section{Graphical abstract}

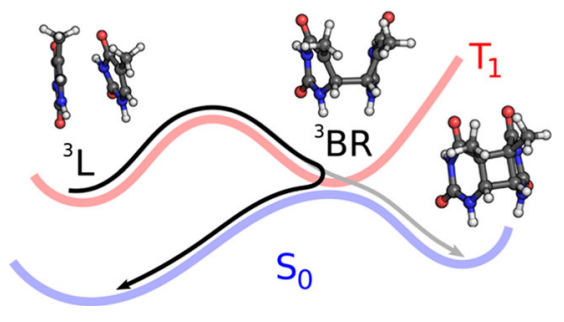

Keywords DNA · Thymine dimerization · Quantum chemical calculations - Non-adiabatic dynamics . Wavefunction analysis - Charge transfer

\section{Introduction}

The formation of cyclobutane thymine $\mathrm{T}\langle\rangle \mathrm{T}$ dimers between two adjacent thymine bases is the most frequent DNA damage under UV radiation [1]. This photolesion, which can take place in both the singlet and triplet manifolds, has been extensively investigated spectroscopically [2-7] and computationally [8-15]. The triplet pathway is a much slower process [7] and exhibits a smaller yield [6, 16] than the singlet mechanism due to inefficient intersystem crossing. As a consequence, this pathway yields very weak spectroscopic signals that preclude unambiguous statements regarding the mechanism [5-7]. In order to enhance the triplet signals, photosensitization is commonly used, increasing the $\mathrm{T}\langle\rangle \mathrm{T}$ dimerization yield [5, 17-19]. This enhancement can also play a role with photosensitizers acting as phototoxic drugs [20]. Photosensitization involves intersystem crossing of a photosensitizer after excitation, transferring its electronic energy to a 


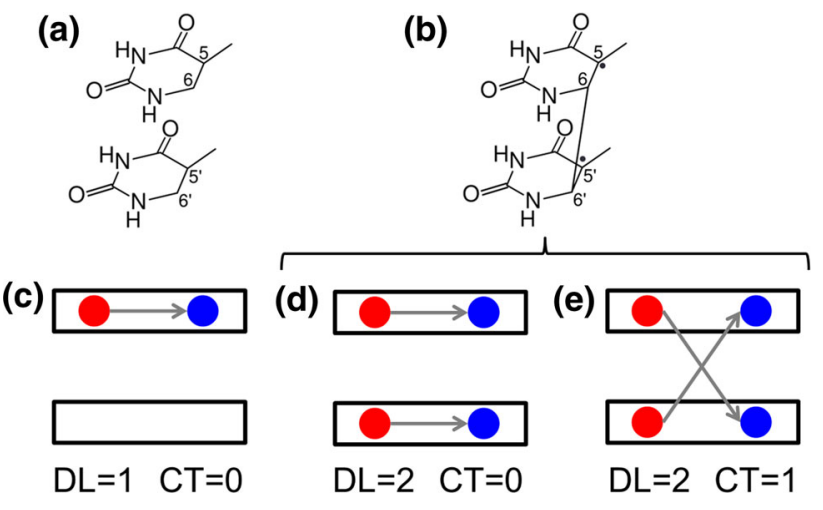

Fig. 1 Chemical formula and electronic arrangement of two thymines for a the local triplet state $\left({ }^{3} \mathrm{~L}\right)$ and $\mathbf{b}$ biradical triplet states $\left({ }^{3} \mathrm{BR}\right)$. Schematic representation of $\mathbf{c}$ a local state, $\mathbf{d}$ a Frenkel exciton state, and $\mathbf{e}$ a charge-resonance state. The black rectangles represent the thymine monomers. The black arrow connects the hole (red circle) and the electron (blue circle) generated after excitation. Delocalization length $(D L)$ and charge-transfer $(C T)$ contribution are also indicated (color figure online)

neighboring thymine, which is then promoted to the lowest triplet state.

Using the photosensitizer 2 -methoxyacetophenone and the dinucleotide TpT, stationary and time-resolved experiments provided two time constants, 22.5 and $62 \mathrm{~ns}$, for the decay of the $\mathrm{TpT}$ in the triplet manifold [5]. These constants have been related to a local triplet state $\left({ }^{3} \mathrm{~L}\right.$, see Fig. 1a, c), which is populated after triplet-triplet energy transfer (TTET) from the photosensitizer, and a biradical triplet state $\left({ }^{3} \mathrm{BR}\right.$, see Fig. 1b, d, e), which can be formed from ${ }^{3}$ L. Quantum chemical calculations [14] suggested that the $\mathrm{T}\langle\rangle \mathrm{T}$ dimerization is triggered by the formation of the biradical intermediate, but the barrierless pathway calculated for the transition from ${ }^{3} \mathrm{~L}$ to ${ }^{3} \mathrm{BR}$ is in conflict with the experimental lifetime of $22.5 \mathrm{~ns}$ assigned to the ${ }^{3} \mathrm{~L}$ species. This conflict is likely caused by the use in the theoretical study of a perfectly stacked geometrical configuration with $C_{\mathrm{s}}$ symmetry, which is hardly achieved in a DNA strand or in a TpT dimer due to the geometrical constraints of the sugar-phosphate backbone. Recent quantum mechanics/molecular mechanics (QM/MM) calculations have found a small barrier of $0.15 \mathrm{eV}$ separating the ${ }^{3} \mathrm{~L}$ and ${ }^{3} \mathrm{BR}$ minima, in better agreement with the experimental lifetime of $22.5 \mathrm{~ns}$ assigned to the ${ }^{3} \mathrm{~L}$ species [15].

An intriguing question in the dimerization process is the character of the ${ }^{3} \mathrm{BR}$ state. Calculations showed that the excited electronic density of ${ }^{3} \mathrm{BR}$ is distributed over the two thymine units [14] and spectroscopic measurements suggested that dimerization involves the participation of delocalized triplet states [18]. However, electronic delocalization over the two monomers can correspond to two different electronic states: (i) a Frenkel exciton, in which two local excitations are coupled (Fig. 1d), or a chargeresonance state, in which two charge-transfer states with charge flow in opposite directions are combined (Fig. 1e) [21]. It has been speculated that the triplet state involved in dimerization could be a charge-transfer state [19], as theoretically predicted for the thymine-thymine 6-4 adduct formation [22]. However, evidence of charge-transfer states for the $\mathrm{T}\langle\rangle \mathrm{T}$ dimerization has never been reported. An additional unsolved mechanistic feature is the reason behind the very low yield of dimerization even when the triplet manifold is forced to be populated after triplet-triplet energy transfer from a photosensitizer.

In this paper, we use quantum chemical calculations, wavefunction analysis, and nonadiabatic surface-hopping molecular dynamics simulations to provide a clear-cut mechanism for the photosensitized thymine dimerization. We study the formation of the ${ }^{3} \mathrm{BR}$ precursor electronic triplet state from the ${ }^{3} \mathrm{~L}$ state and identify the nature of these species in terms of electronic delocalization and charge-transfer character. Furthermore, we offer a rationale for the factors behind the small quantum yield of the reaction.

\section{Results and discussion}

The first step of our study is to select the level of theory for the electronic-structure calculations, especially for the nonadiabatic surface-hopping dynamics simulations. We commence by computing the lowest-energy band of the density of triplet states, which involves the $T_{1}$ and $T_{2}$ electronic states, of a thymine-thymine stacked pair embedded in a solvated single strand $(\mathrm{dT})_{12}$. Triplet excitation energies were calculated with an electrostatic QM/ MM [23] scheme where the two nucleobases in the middle of the strand were described by multistate complete active space second-order perturbation [24] (MS-CASPT2) theory and the rest of the system by a force field $[25,26]$. The QM region was also described by state-average complete active space self-consistent field (SA-CASSCF) [27] to investigate whether dynamical correlation is necessary to describe the lowest-energy triplet band. In addition, the MSCASPT2/MM calculations have been performed employing two different active spaces, namely $(4,4)$ and $(8,8)$. The first one only includes the four $\pi$ orbitals and the four electrons involved in the dimerization reaction (orbitals $\pi_{3}$, $\pi_{4}, \pi_{5}^{*}$, and $\pi_{6}^{*}$ in Fig. 9). The second active space has two additional electrons and two additional $\pi$ orbitals for each nucleobase. The calculations were performed on an ensemble of 250 geometries taken from a previous groundstate QM/MM molecular dynamics simulation [12]. The density-of-states bands computed at the different levels of theory are plotted in Fig. 2a. The MS-CASPT2(8,8)/MM 


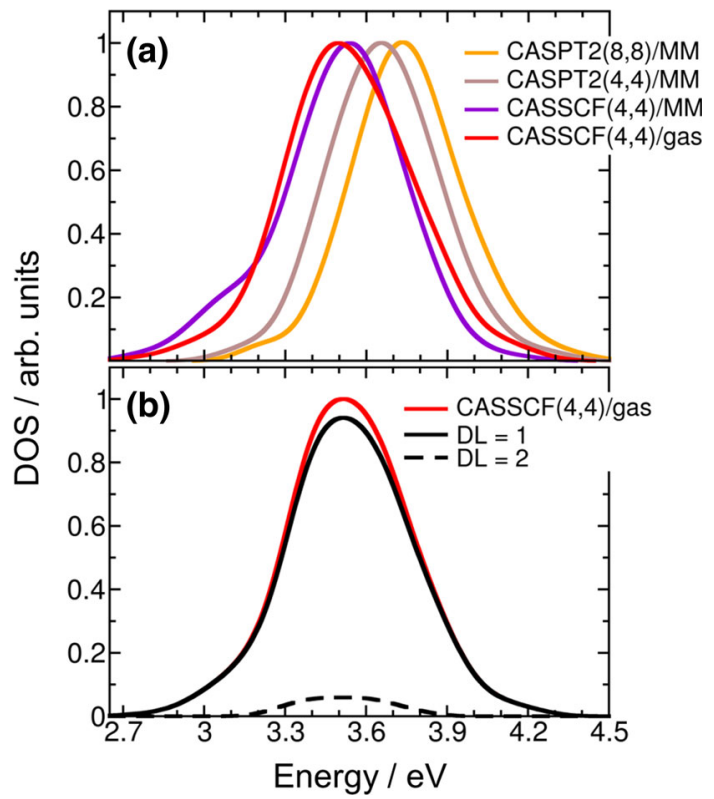

Fig. 2 a Lowest-energy band of density of triplet states for the thymine-thymine stacked pair embedded in a solvated single strand $(\mathrm{dT})_{12}$ computed at MS(3)-CASPT2 $(8,8) / \mathrm{MM}$, MS(3)-CASPT2 $(4,4) /$ MM, SA(3)-CASSCF(4,4)/MM, and SA(3)-CASSCF(4,4)/gas phase levels of theory and for the thymine-thymine stacked pair in the gas phase computed at SA-CASSCF level of theory. b Delocalization length $(D L)$ decomposition of the $\operatorname{SA}(3)-\operatorname{CASSCF}(4,4)$ density of triplet states of the thymine-thymine stacked pair in the gas phase

band is blue shifted by only $0.08 \mathrm{eV}$ with respect to the MS-CASPT2 $(4,4) / \mathrm{MM}$ one. This means that the smaller active space is enough to describe most of the static correlation. The small energy difference of $0.12 \mathrm{eV}$ between MS-CASPT2 $(4,4) / \mathrm{MM}$ and SA-CASSCF(4,4)/MM shows that a correct qualitative picture can be obtained without including dynamical correlation in the calculation. The electrostatic effect of the solvated DNA environment in the triplet excited states is small. This can be seen by comparing the SA-CASSCF $(4,4) / \mathrm{MM}$ and SA-CASSCF $(4,4) /$ gas phase bands, whose energy maxima differ only by $0.04 \mathrm{eV}$. Overall, the energy difference between the highest level of theory [MS-CASPT2 $(8,8) / \mathrm{MM}$ ] and the lowest level of theory [SA-CASSCF $(4,4)$ in the gas phase] employed here is $0.24 \mathrm{eV}$. Therefore, based on these results at the Franck-Condon region, SA-CASSCF calculations in the gas phase seem to be suitable to describe the lowest-energy triplet states of the thymine dimer embedded in a DNA strand.

The first step of the reaction is the population of $T_{1}$ after TTET. The character of the $T_{1}$ state can either be ${ }^{3} \mathrm{~L}$ or ${ }^{3} \mathrm{BR}$ depending on its electronic configuration (see Fig. 1). For most of the geometries within the Franck-Condon region, it is expected that the $T_{1}$ electronic state corresponds to the locally excited configuration ${ }^{3} \mathrm{~L}$ as the relatively large rise distance $(3.5 \AA)$ between stacked nucleobases in DNA strands mostly precludes the direct formation of ${ }^{3} \mathrm{BR}$ state, where the $\mathrm{C}_{6}-\mathrm{C}_{6}{ }^{\prime}$ bond is already preformed. The excited electronic density in ${ }^{3} \mathrm{~L}$ is completely located at one of the thymine nucleobases (Fig. 1c), while ${ }^{3} \mathrm{BR}$ has the spin density equally distributed over the ethylenic $\mathrm{C}_{5}$ and $\mathrm{C}_{5}{ }^{\prime}$ atoms of both thymine bases (Fig. 1d, e). Since the $\mathrm{C}_{6}-\mathrm{C}_{6}{ }^{\prime}$ bond is already preformed in the ${ }^{3} \mathrm{BR}$ species, it is likely that the $\mathrm{T}\langle\rangle \mathrm{T}$ dimerization is triggered by the formation of the biradical intermediate, as suggested in the literature $[5,14]$.

Even if the $\mathrm{C}_{6}-\mathrm{C}_{6}{ }^{\prime}$ bond is not preformed within the Franck-Condon region, we found it interesting to investigate whether any initial geometrical configuration presents ${ }^{3}$ BR character. To this aim, we analyzed the electronic transition density $[21,28,29]$ of the triplet states that compose the density of states, from which the delocalization length $(D L)$, defined as the number of nucleobases involved in the excitation process [30], was computed. For ${ }^{3} \mathrm{~L}$, the excitation is localized in only one of the thymine bases $(D L=1)$, while in ${ }^{3} \mathrm{BR}$ both thymine monomers are involved in the excitation $(D L=2)$. Figure $2 \mathrm{~b}$ shows the calculated density of triplet states in the gas phase decomposed by delocalization length. We find that the lowest-energy triplet band is mainly composed by local excitations ${ }^{3} \mathrm{~L}$, while the contribution of excitations delocalized over the two monomers is very small. Since the photosensitizer employed in the experiments [5] was initially excited at $\sim 4 \mathrm{eV}$, the calculated states composing this band (between 2.7 and $4.4 \mathrm{eV}$ ) are the only ones energetically accessible by triplet-triplet energy transfer. Unequivocally, most of the states populated at the FranckCondon region are locally excited states, i.e. correspond to the ${ }^{3} \mathrm{~L}$ triplet state.

After having established that the ${ }^{3} \mathrm{~L}$ state is initially populated, in agreement with spectroscopic measurements, the next step is the formation of the ${ }^{3} \mathrm{BR}$ state. Figure $3 \mathrm{a}$ shows the MS-CASPT2 energies of the $S_{0}, T_{1}$, and $T_{2}$ state in a static scan from the ${ }^{3} \mathrm{~L}$ state to the ${ }^{3} \mathrm{BR}$ minimum and from the ${ }^{3} \mathrm{BR}$ minimum to the dimer. In qualitative agreement with the barrier obtained in Ref. [15], a barrier of $0.27 \mathrm{eV}$ separates the ${ }^{3} \mathrm{~L}$ and the ${ }^{3} \mathrm{BR}$ minima in $T_{1}$. This energy barrier agrees very well with the barrier of $0.30 \mathrm{eV}$ that is obtained by using the Arrhenius equation at a temperature of $300 \mathrm{~K}$ and using the experimental deactivation time of $22 \mathrm{~ns}$ [5], despite the approximations taken. The relatively large energy barrier is likely the first reason that explains the low yield of the reaction as in many cases the system has enough time to return to the ground state by intersystem crossing before overcoming the barrier.

The electronic wavefunction of $T_{1}$ along the pathway between ${ }^{3} \mathrm{~L}$ and ${ }^{3} \mathrm{BR}$ is analyzed in Fig. 3b, c. Specifically, the delocalization length $(D L)$ and the charge-transfer fraction were computed from the electronic transition 
Fig. 3 Variation of $\mathbf{a}, \mathbf{d}$ the potential energy of $S_{0}, T_{1}$, and $T_{2}$, and $\mathbf{b}$, e charge-transfer $(C T)$ contribution and $\mathbf{c}, \mathbf{f}$ the delocalization length $(D L)$ of $T_{1}$ along a linearly interpolated pathway along the reaction coordinate (average of the $\mathrm{C}_{6}-$ $\mathrm{C}_{6}{ }^{\prime}$ and $\mathrm{C}_{5}-\mathrm{C}_{5}{ }^{\prime}$ bond lengths) connecting the initial ${ }^{3} \mathrm{~L}$ structure with the ${ }^{3} \mathrm{BR}$ minimum and continuing from there to the thymine dimer. The calculations were carried out for the gas phase employing MSCASPT2 $(4,4)$ and SACASSCF $(4,4)$ levels of theory

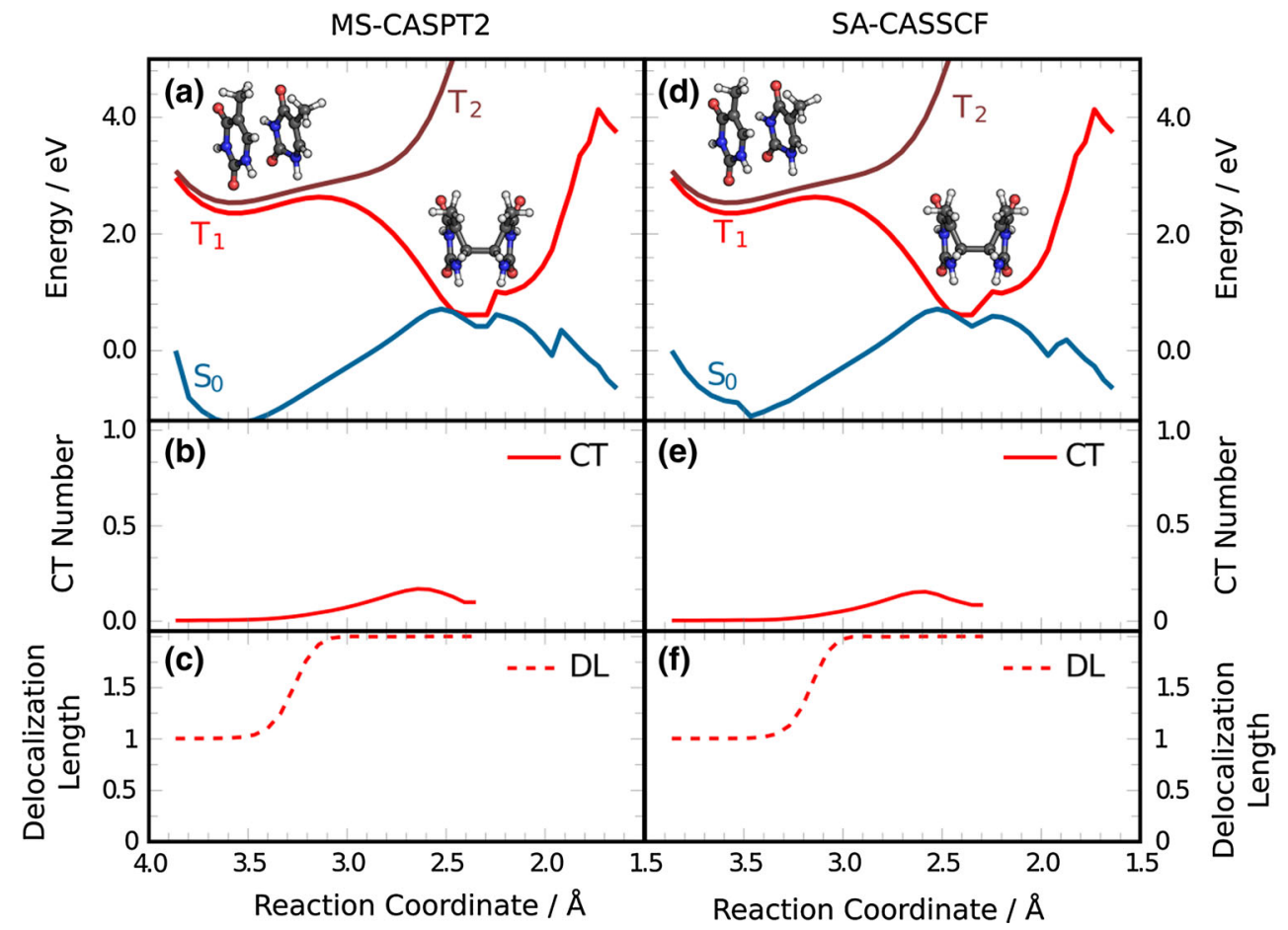

density [21, 28, 29]. The delocalization length clearly shows that the dimer is in a locally excited state $(D L=1)$ before the barrier and, after overcoming the barrier, it evolves towards the ${ }^{3} \mathrm{BR}$ excited state $(D L=2)$. This ${ }^{3} \mathrm{BR}$ excited state can be a Frenkel exciton state or a chargeresonance state (recall Fig. 1d, e). Due to the small separation between both thymine monomers at the ${ }^{3} \mathrm{BR}$ minimum, the formation of charge-transfer states, favoured by orbital-overlap interactions [31], is possible. Therefore, the ${ }^{3} \mathrm{BR}$ state could acquire charge-resonance character along the dimerization pathway. The solid line in Fig. 3b unambiguously shows that the charge-transfer contribution is very small along the path that connects ${ }^{3} \mathrm{~L}$ with ${ }^{3} \mathrm{BR}$. This demonstrates that ${ }^{3} \mathrm{BR}$ is mainly a Frenkel exciton state. Only in the region near to the ${ }^{3} \mathrm{BR} / S_{0}$ crossing the charge-transfer contribution is around 0.15 , indicating that the Frenkel state acquires a small degree of charge-transfer character. This conclusion is in contrast to the hypothesis put forward in Ref. [19], claiming that charge-transfer triplet states could be present in the $\mathrm{T}\langle\rangle \mathrm{T}$ dimerization. Our calculations clearly demonstrate that the precursor electronic state leading to dimerization is a Frenkel exciton state and not a charge-transfer state. Recent theoretical calculations predicted that $\mathrm{T}\langle\rangle \mathrm{T}$ dimerization in the singlet manifold is also mediated by an exciton intermediate [8]. Figure $3 \mathrm{~d}-\mathrm{f}$ shows the same energy scan and wavefunction analysis computed at SA-CASSCF level. Since the energy and character of the states are very similar to the ones obtained by MS-CASPT2, as was also the case for the density of states computed at the Franck-Condon region, the subsequent gas-phase dynamics simulations are performed using SA-CASSCF for the electronic-structure calculations.

After the formation of the ${ }^{3} \mathrm{BR}$ species, the system is trapped in the ${ }^{3} \mathrm{BR}$ minimum (recall Fig. 3a). This minimum coincides with the crossing point with the ground state $S_{0}$. Dimerization takes place only when the appropriate region of the $S_{0}$ potential is populated after intersystem crossing from $T_{1}$. As the experimental [5] decay time constant is $62 \mathrm{~ns}$ for ${ }^{3} \mathrm{BR}$, the radiationless decay to the ground state is a very slow process. Once in the ground state, the system can dimerize or return to the reactant region without causing damage. The experimentally determined dimerization yield is only 4\% [5]. In order to determine the factors that govern this low yield, we have sampled the ${ }^{3} \mathrm{BR}$ minimum of $T_{1}$ for at least $100 \mathrm{fs}$ with non-adiabatic surface hopping molecular dynamics simulations in the gas phase using the SHARC code [32].

As expected, none of the trajectories that sampled the $T_{1}$ minimum showed intersystem crossing to the ground state during $100 \mathrm{fs}$. This is because the spin-orbit coupling around the ${ }^{3} \mathrm{BR} / S_{0}$ crossing, computed for one of the trajectories as the averaged spin-orbit coupling of 100 snapshots, is merely $1 \mathrm{~cm}^{-1}$. Since the intersystem crossing rate depends on the spin-orbit coupling [33], the system can survive in the $T_{1}$ minimum for a long time (see Fig. 4 for an example trajectory), in agreement with the large experimental deactivation time of $62 \mathrm{~ns}$ [5] and previous calculations [14]. In order to simulate the last step of $\mathrm{T}\langle\rangle \mathrm{T}$ dimerization, 32 snapshots from the trajectories trapped in 


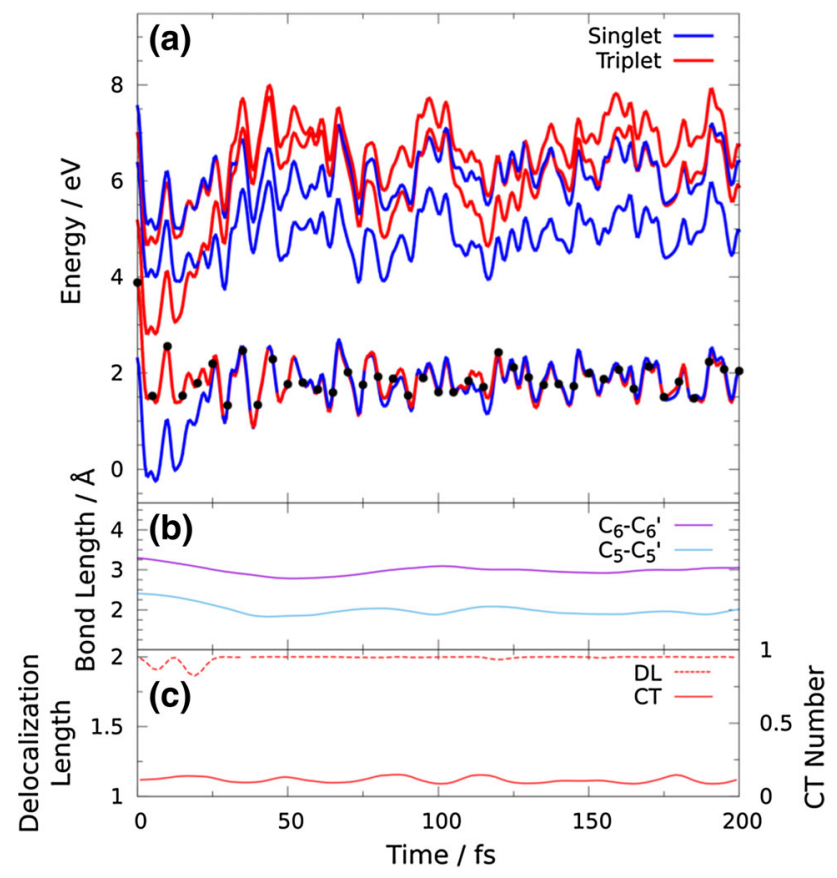

Fig. 4 Time evolution of a energy levels, $\mathbf{b}$ the $\mathrm{C}_{5}-\mathrm{C}_{5}{ }^{\prime}$ and $\mathrm{C}_{6}-\mathrm{C}_{6}{ }^{\prime}$ distances, and $\mathbf{c}$ the delocalization length and charge-transfer $(C T)$ contribution for a trajectory trapped in the minimum of $T_{1}$

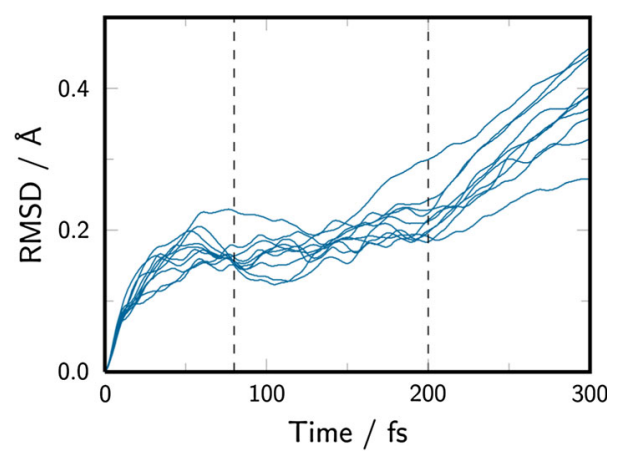

Fig. 5 Root-mean squared displacement (RMSD) of the nonadiabatic dynamics trajectories running in the $T_{1}$ state. Dashed lines indicate the area from which the geometries were randomly chosen to be manually placed to the ground state

the $T_{1}$ state were chosen based on a combination of random selection and an ${ }^{3} \mathrm{BR} / S_{0}$ energy gap smaller than $0.15 \mathrm{eV}$. At these snapshots, the molecules were manually placed in the ground state, and the dynamics was continued. The selected geometries show an average ${ }^{3} \mathrm{BR} / S_{0}$ energy gap of $0.07 \mathrm{eV}$ and were taken from the time region of 80-200 fs, based on the root mean squared displacement (RMSD) of the trajectories running in the $T_{1}$ state (see Fig. 5). The RMSD shows that at times shorter than 80 fs the geometry of the dimer is not equilibrated and at times longer than $200 \mathrm{fs}$ the nucleobases go apart due to the lack of the sugar-phosphate backbone. Only 5 of these 32 trajectories lead to dimerization, while 27 trajectories returned to the

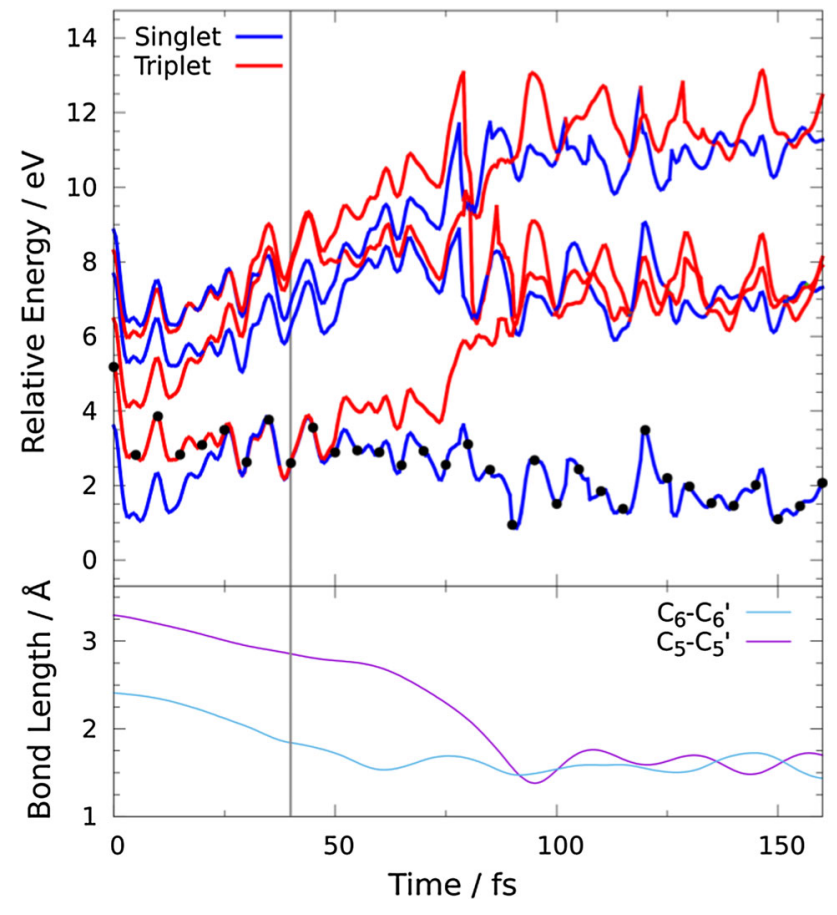

Fig. 6 Time evolution of $\mathbf{a}$ energy levels and $\mathbf{b}$ the $\mathrm{C}_{5}-\mathrm{C}_{5}{ }^{\prime}$ and $\mathrm{C}_{6}-$ $\mathrm{C}_{6}{ }^{\prime}$ distances for a reactive trajectory manually placed in the ground state close to the ${ }^{3} \mathrm{BR} / S_{0}$ crossing point. The vertical grey line indicates the moment at which the trajectory is transferred to the $S_{0}$

reactant region. Figure 6 displays an example reactive trajectory, in which, first, the $\mathrm{C}_{6}-\mathrm{C}_{6}{ }^{\prime}$ bond formation is completed after $60 \mathrm{fs}$, and then, the $\mathrm{C}_{5}-\mathrm{C}_{5}{ }^{\prime}$ bond is formed after additional $40 \mathrm{fs}$. The low number of reactive trajectories qualitatively agrees with the low experimental yield of $4 \%$ obtained from spectroscopic measurements [5]. However, due to the small number of trajectories employed here, we cannot make any comments on the statistics of the reaction.

The low dimerization yield is rationalized by analyzing the space of coordinates and velocities (phase space) at the moment of the ${ }^{3} \mathrm{BR} / S_{0}$ transition $[34,35]$. Note that these ${ }^{3} \mathrm{BR} / S_{0}$ transitions are approximated by the selection process described above. The relevant internal coordinates that drive the reaction are the $\mathrm{C}_{5}-\mathrm{C}_{5}{ }^{\prime}$ and $\mathrm{C}_{6}-\mathrm{C}_{6}{ }^{\prime}$ distances, and the relevant velocities are those of the atoms involved in these distances. In Fig. $7 \mathrm{a}, \mathrm{b}$, the values of the $\mathrm{C}_{6}-\mathrm{C}_{6}{ }^{\prime}$ and $\mathrm{C}_{5}-\mathrm{C}_{5}{ }^{\prime}$ distances and average angle formed by the velocity vectors of the atoms $\mathrm{C}_{5}$ and $\mathrm{C}_{5}{ }^{\prime}$ with the $\mathrm{C}_{5}-\mathrm{C}_{5}{ }^{\prime}$ vector $\left(\theta_{5}\right.$ and $\left.\theta_{5}{ }^{\prime}\right)$, and by the velocity vectors of the atoms $\mathrm{C}_{6}$ and $\mathrm{C}_{6}{ }^{\prime}$ with the $\mathrm{C}_{6}-\mathrm{C}_{6}{ }^{\prime}$ vector $\left(\theta_{6}\right.$ and $\left.\theta_{6}{ }^{\prime}\right)$, are plotted at the moment of the ${ }^{3} \mathrm{BR} / S_{0}$ transition. Those trajectories that underwent dimerization are represented by green pentagons. Only when the $\mathrm{C}_{6}-\mathrm{C}_{6}{ }^{\prime}$ and $\mathrm{C}_{5}-\mathrm{C}_{5}{ }^{\prime}$ distances are lower than 2.1 and $3.0 \AA$, respectively, dimerization takes place. In addition, the $\mathrm{C}$ atoms of each monomer also need to move towards each other with a large degree of 

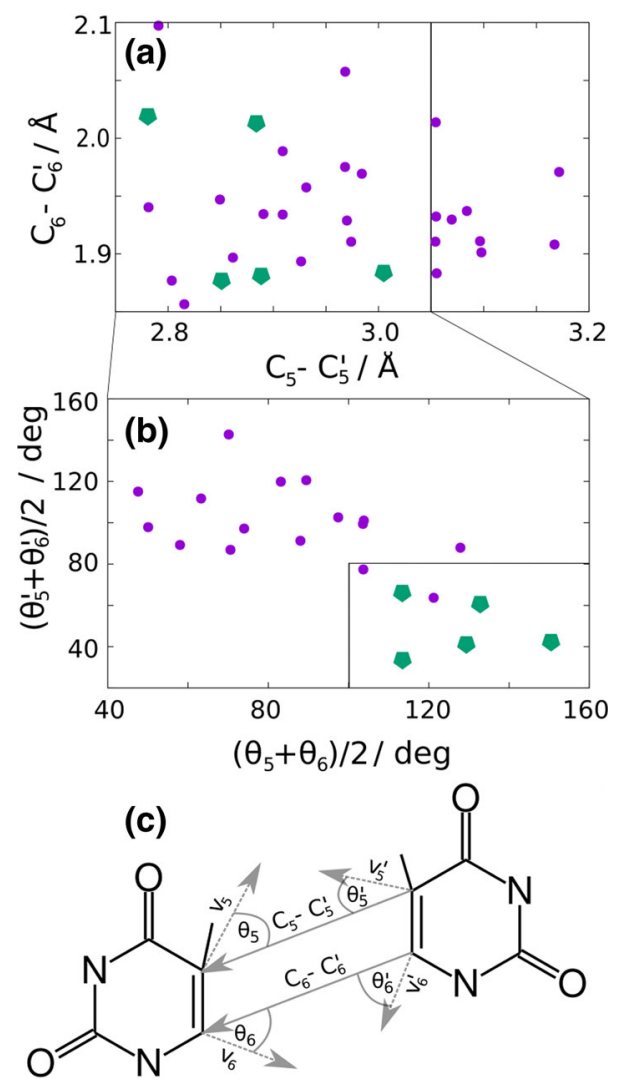

Fig. 7 a $\mathrm{C}_{5}-\mathrm{C}_{5}{ }^{\prime}$ and $\mathrm{C}_{6}-\mathrm{C}_{6}{ }^{\prime}$ distances, $\mathbf{b}$ average angles formed between the distances $\mathrm{C}_{5}-\mathrm{C}_{5}{ }^{\prime}$ and $\mathrm{C}_{6}-\mathrm{C}_{6}{ }^{\prime}$ and the velocities at the atoms $\mathrm{C}_{5}$ and $\mathrm{C}_{6}$ at the moment of the ${ }^{3} \mathrm{BR} \rightarrow S_{0}$ transition for 32 trajectories. c Definition of distances and angles employed in the analysis. The lines across a-c indicate that the next panel is a subset of the data points marked in the box of the previous panel. The green pentagons indicate trajectories undergoing dimerization (color figure online)

directionality, as indicated by the restricted values of the $\theta$ angles [larger than $100^{\circ}$ for $\left(\theta_{5}+\theta_{6}\right) / 2$ and smaller than $80^{\circ}$ for $\left.\left(\theta_{5}{ }^{\prime}+\theta_{6}{ }^{\prime}\right) / 2\right]$. Therefore, although the phase space of the system is very wide, due to the large number of degrees of freedom of the system, only the population of a very small region of the phase space induces dimerization. This is the second reason that is responsible for the very small dimerization yield.

\section{Conclusion}

In summary, based on our theoretical results and previous experiments [5], we propose the following stepwise mechanism for the photosensitized $\mathrm{T}\langle\rangle \mathrm{T}$ dimerization, schematically represented in Fig. 8. First, the locally excited triplet state ${ }^{3} \mathrm{~L}$ of thymine is populated after triplettriplet energy transfer from a photosensitizer [step (i) in Fig. 8]. Then, the system vibrationally relaxes to the ${ }^{3} \mathrm{~L}$ minimum where it stays for $22.5 \mathrm{~ns}$ (ii). After overcoming an energy barrier of ca. $0.3 \mathrm{eV}$ (iii), a biradical intermediate ${ }^{3} \mathrm{BR}$ with a lifetime of $62 \mathrm{~ns}$ is generated within a region that crosses with the electronic ground state. The populated triplet state of the intermediate species is a Frenkel exciton with a small degree of charge-transfer character. Finally, the system undergoes intersystem crossing from $T_{1}$ to the ground state (iv), from where it dimerizes with a very small yield, i.e. returning to the initial reactant geometries consisting of two separated thymines in most events (v) due to the tight phase-space restrictions that the system needs to satisfy at the moment of the $T_{1} \rightarrow S_{0}$ transition.

\section{Methods}

\section{QM/MM calculation of density of states}

The density of states associated to the lowest-energy triplet band of the thymine dimer embedded in a solvated single strand $(\mathrm{dT})_{12}$ and in the gas phase was computed. First, a isothermal-isobaric ensemble (NPT) classical molecular dynamics simulation for solvated (dT) 12 was evolved at $300 \mathrm{~K}$ for $20 \mathrm{~ns}$ using the ff14SB [26] and TIP3P [25] force fields to describe DNA and water, respectively. The classical simulation was run with the graphical processing unit (GPU) module pmemd [36] implemented in the Amber14 package [37]. Then, the last snapshot of the classical simulation was taken as the starting one for running quantum mechanics/molecular mechanics (QM/MM) molecular dynamics simulations in the NPT ensemble for $10 \mathrm{ps}$. The two nucleobases in the middle of the strand were described by the B3LYP functional [38] with D3 dispersion correction [39] and the $6-31 \mathrm{G}^{*}$ basis set $[40,41]$ using the GPU-based code TeraChem1.9 [42, 43] through the interface to external QM programs implemented in Amber14 [37]. More computational details about the molecular dynamics simulations can be found in Ref. [12].

An ensemble of 250 equidistant snapshots was selected from the last $5 \mathrm{ps}$ of the QM/MM molecular dynamics simulation. For each snapshot, the electronic excitation energies of the lowest 3 triplet states were computed using an electrostatic embedding QM/MM scheme. The two nucleobases in the middle of the $(\mathrm{dT})_{12}$ strand are described by state-averaged complete active space self-consistent field [27] (SA-CASSCF) using the cc-pVDZ basis set $[44,45]$, and also by multistate complete active space second-order perturbation (MS-CASPT2) [24] with the same basis set. To minimize the effect of intruder states the level-shift approach was applied with a real-valued shift of 0.3 a.u. The IPEA shift was set to zero, as it is recommended for organic chromophores [46]. The rest of the 
Fig. 8 Proposed mechanism of photosensitized $\mathrm{T}\langle\rangle \mathrm{T}$ dimerization in the triplet state. (i) Triplet-triplet energy transfer (TTET) from the photosensitizer (PS) to thymine-thymine, (ii) vibrational relaxation (VR) in $T_{1}$, (iii) ${ }^{3} \mathrm{BR}$ formation by overcoming an energy barrier, (iv) intersystem crossing (iv), and (v) formation of thymine dimer or return to the FranckCondon (FC) region in the electronic ground state

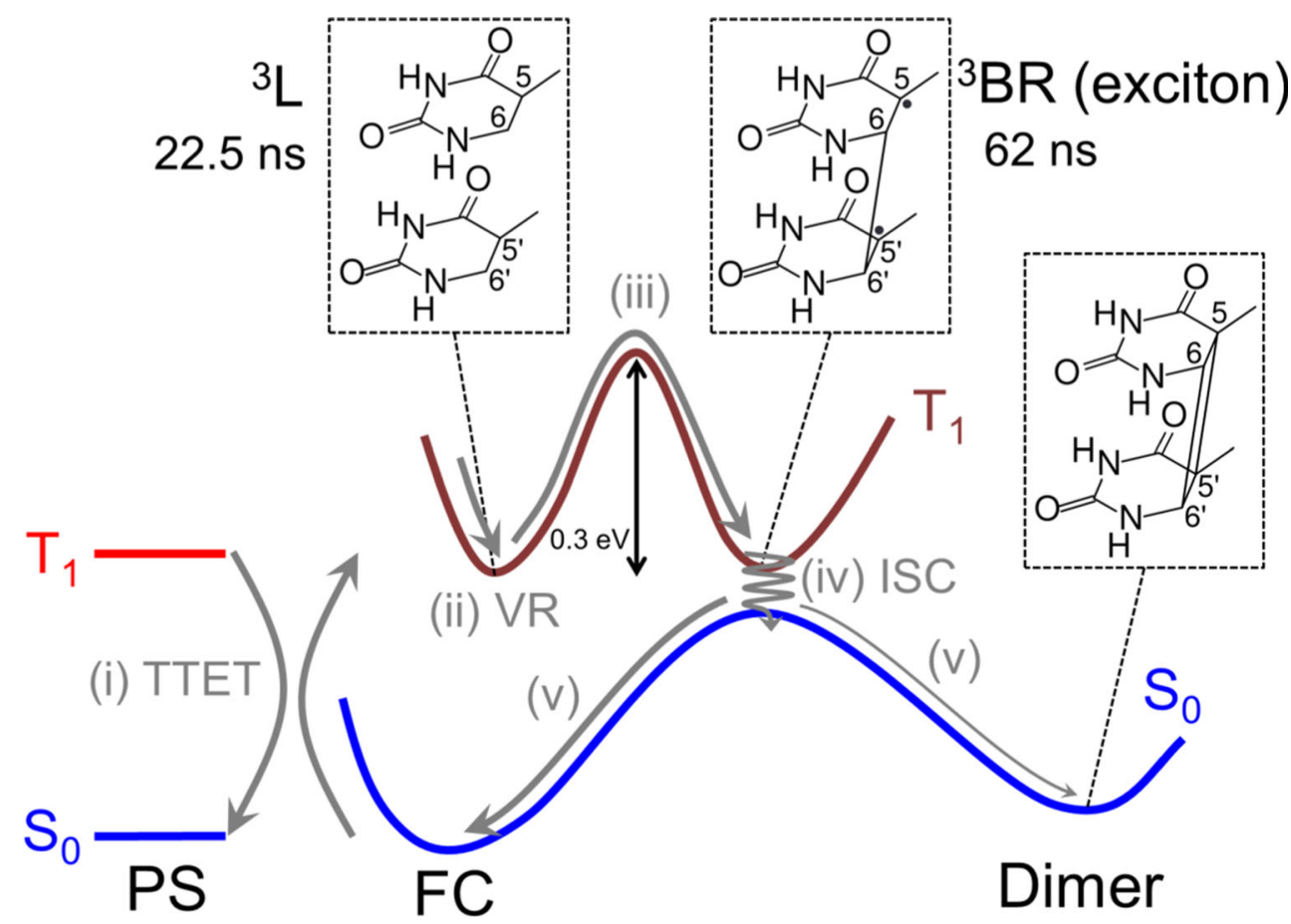

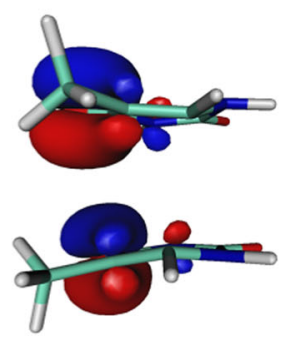

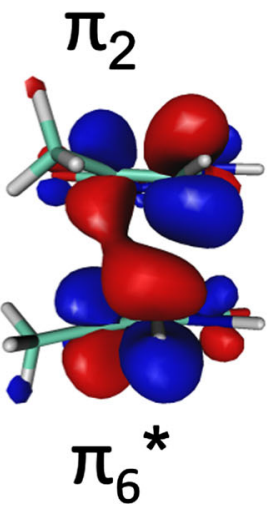

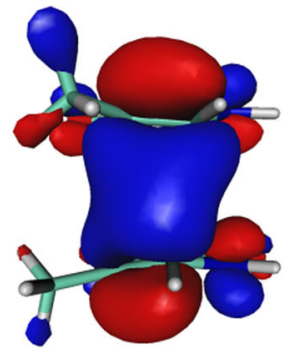

$\pi_{3}$

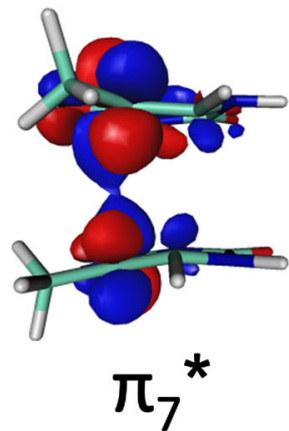

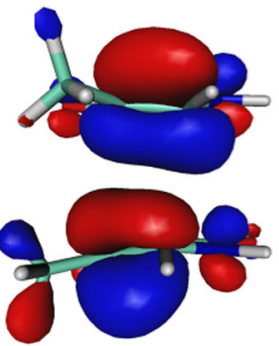

$\pi_{4}$

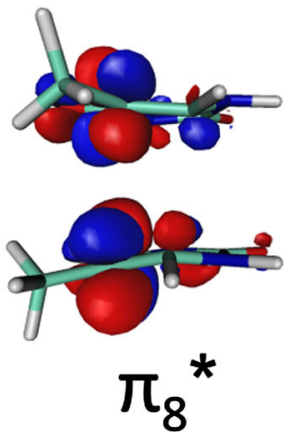

DNA strand and the water molecules were described by a force field [25, 26]. In addition, the calculations were performed in the gas phase by removing the environment from the 250 snapshots. The two active spaces considered in the calculations consist of 8 electrons in 8 orbitals and of 4 electrons in 4 orbitals (see Fig. 9). These calculations were carried out with MOLCAS 8 [28, 47]. The resulting excitation energies were convoluted with Gaussian functions with a full width at half maximum of $0.20 \mathrm{eV}$. The intensity of the bands was scaled to unity. In addition, all electronic triplet states in the gas phase were classified as local states $\left({ }^{3} \mathrm{~L}\right)$ or biradical states $\left({ }^{3} \mathrm{BR}\right)$ according to the electronic delocalization length, defined as the number of nucleobases involved in the excitation, computed from the electronic transition density $[21,29,30]$. For ${ }^{3} \mathrm{~L}$, the excitation is localized in only one of the thymine bases 
$(D L=1)$, while in ${ }^{3} \mathrm{BR}$ both thymine monomers are involved in the excitation $(D L=2)$. The MS-CASPT2/ $\mathrm{MM}$, SA-CASSCF/MM, and SA-CASSCF/gas phase density of states and the delocalization-length decomposition of the gas-phase band are plotted in Fig. 2.

\section{Energy scan in the $T_{1}$ potential energy surface}

The static calculations for the potential energy scan (Fig. 3), which goes from the Franck-Condon region to dimer formation, were carried out using MS-CASPT2 (Fig. 3a) and SA-CASSCF (Fig. 3d) with the previously described $(4,4)$ active space and the cc-pVDZ basis set. The ${ }^{3} \mathrm{~L}$ geometry of the Franck-Condon region was taken from the ground state QM/MM molecular dynamics simulation explained above. Specifically, for every of the 30 snapshots whose vertical energy for $T_{1}$ is below $3.5 \mathrm{eV}$, which corresponds to the maxima of lowest-energy band of the density of states, the static scan was performed. Only the scan with the lowest energy barrier in the $T_{1}$ state, which tries to mimic a minimum-energy path calculation, is shown in Fig. 3. The geometry at the crossing point between $S_{0}$ and $T_{1}$ was taken from Ref. [14]. The energies of the two lowest triplet states were computed along a linearly interpolated pathway between both geometries. From the crossing point a second linearly interpolated pathway was connected to the dimer structure, which was taken from our previous work [12]. Moreover, the chargetransfer contribution and delocalization length were also computed along the interpolated pathway using both MSCASPT2 (Fig. 3b, c) and SA-CASSCF (Fig. 3e, f) [21, 29].

\section{Non-adiabatic molecular dynamics simulations}

Non-adiabatic molecular dynamics simulations were run to sample the $T_{1} / S_{0}$ degeneracy region, in which the $T_{1}$ state presents biradical character. Therefore, an arbitrary initial geometry was built with interatomic $\mathrm{C}_{5}-\mathrm{C}_{5}{ }^{\prime}$ and $\mathrm{C}_{6}-\mathrm{C}_{6}{ }^{\prime}$ distances of 3.13 and $2.45 \AA$, respectively. From this geometry 1000 initial conditions (coordinates and velocities) were generated from a zero-Kelvin Wigner distribution [48] based on ground-state frequencies calculated at second-order Møller-Plesset (MP2) perturbation theory [49] using the cc-pVTZ basis set [44] implemented in MOLPRO [50]. From these 1000 initial conditions, 25 were randomly selected to run dynamics on. All trajectories were initially excited to the $T_{1}$ state and ran for at least 100 fs or until they left the $T_{1} / S_{0}$ degeneracy region. As the dynamics starts at a close thymine-thymine distance, it was assumed that the reaction is already in progress at the start of the dynamics. Therefore, the initial velocities of all trajectories were modified so that the center of mass of each monomer moves towards each other at a velocity corresponding to the thermal energy $\left(k_{\mathrm{B}} T\right)$ at a temperature of $298 \mathrm{~K}$.

From the trajectories running in the degeneracy region, 32 geometries were chosen based on a combination of random selection as well as an ${ }^{3} \mathrm{BR} / S_{0}$ energy gap smaller than $0.15 \mathrm{eV}$ and continued to run on the ground state potential energy surface. This approach was necessary as none of the trajectories that ran in $T_{1}$ hopped to the ground state during their simulation time. The geometries and velocities for the new trajectories running in $S_{0}$ were taken from the point where they manually hopped from the parent trajectory, and the electronic coefficients were adapted to put the population on the ground state.

The dynamics simulations were carried out using the ab initio molecular dynamics program SHARC (surface hopping including arbitrary couplings) [32, 51], which uses a modification of the Tully surface hopping method [52] allowing for treating both singlet and triplet states on the same footing. The time step used for the nuclear motion was $0.5 \mathrm{fs}$, and the time step for the integration of the timedependent electronic Schrödinger equation was $0.02 \mathrm{fs}$. All electronic structure properties (energies, gradients, and couplings) were calculated at the SA-CASSCF level of theory using the above described $(4,4)$ active space and the cc-pVDZ basis set. For both the singlet and the triplet state calculations, 3 states were averaged with equal weights each. The non-adiabatic couplings were calculated from the wavefunction overlaps by using a local-diabatization scheme [53]. Additionally, this procedure monitors the wavefunction phase and makes sure that it is maintained throughout the dynamics [54]. Moreover, the Persico decoherence correction [55], with a decoherence parameter of 0.1 a.u. was employed. To save computational time, the gradients of not-populated states were only calculated when their energy was within $0.5 \mathrm{eV}$ of the currently populated state. This procedure is in accordance with previous studies showing that higher lying states only have a minimal effect on the potential of the populated states [32].

Acknowledgements Open access funding provided by University of Vienna. CR gratefully acknowledges the University of Vienna within the uni:docs programme for financial support. CR also thanks Chemical Monthly, Springer, the Austrian Academy of Sciences, and the Austrian Chemical Society (GÖCH) for a mobility fellowship. The authors thank Felix Plasser for fruitful discussions. The computational results have been achieved in part using the Vienna Scientific Cluster (VSC).

Open Access This article is distributed under the terms of the Creative Commons Attribution 4.0 International License (http://crea tivecommons.org/licenses/by/4.0/), which permits unrestricted use, distribution, and reproduction in any medium, provided you give appropriate credit to the original author(s) and the source, provide a link to the Creative Commons license, and indicate if changes were made. 


\section{References}

1. Beukers R, Eker APM, Lohman PHM (2008) DNA Repair 7:530

2. Schreier WJ, Kubon J, Regner N, Haiser K, Schrader TE, Zinth W, Clivio P, Gilch P (2009) J Am Chem Soc 131:5038

3. Schreier WJ, Schrader TE, Koller FO, Gilch P, Crespo-Hernández CE, Swaminathan VN, Carell T, Zinth W, Kohler B (2007) Science 315:625

4. Desnous C, Babu BR, Moriou C, Mayo JUO, Favre A, Wengel J, Clivio P (2008) J Am Chem Soc 130:30

5. Liu L, Pilles BM, Gontcharov J, Bucher DB, Zinth W (2016) J Phys Chem B 120:292

6. Pilles BM, Bucher DB, Liu L, Clivio P, Gilch P, Zinth W, Schreier WJ (2014) J Phys Chem Lett 5:1616

7. Kwok WM, Ma C, Phillips DL (2008) J Am Chem Soc 130:5131

8. Conti I, Martínez-Fernández L, Esposito L, Hofinger S, Nenov A, Garavelli M, Improta R (2017) Chem Eur J 23:15177

9. Boggio-Pasqua M, Groenhof G, Schäfer LV, Grubmüler H, Robb MA (2007) J Am Chem Soc 129:10996

10. Blancafort L, Migani A (2007) J Am Chem Soc 129:14540

11. Spata VA, Lee W, Matsika S (2016) J Phys Chem Lett 7:976

12. Rauer C, Nogueira JJ, Marquetand P, González L (2016) J Am Chem Soc 138:15911

13. Mendieta-Moreno JI, Trabada DG, Mendieta J, Lewis JP, Gómez-Puertas P, Ortega J (2016) J Phys Chem Lett 7:4391

14. Climent T, González-Ramírez I, González-Luque R, Merchán M, Serrano-Andrés L (2010) J Phys Chem Lett 1:2072

15. Wang H, Chen X (2017) Molecules 22:60

16. Banyasz A, Douki T, Improta R, Gustavsson T, Onidas D, Vayá I, Perron M, Markovitsi D (2012) J Am Chem Soc 134:14834

17. Cuquerella MC, Lhiaubet-Vallet V, Bosca F, Miranda MA (2011) Chem Sci 2:1219

18. Miro P, Lhiaubet-Vallet V, Marin ML, Miranda MA (2015) Chem Eur J 21:17051

19. Douki T, Bérard I, Wack A, Andrä S (2014) Chem Eur J 20:5787

20. Moore DE (2002) Drug Saf 25:345

21. Plasser F, Lischka H (2012) J Chem Theory Comput 8:2777

22. Giussani A, Serrano-Andrés L, Merchán M, Roca-Sanjuán D, Garavelli M (2013) J Phys Chem B 117:1999

23. Melaccio F, Olivucci M, Lindh R, Ferré N (2011) Int J Quantum Chem 111:3339

24. Finley J, Malmqvist PA, Roos BO, Serrano-Andrés L (1998) Chem Phys Lett 288:299

25. Jorgensen WL, Chandrasekhar J, Madura JD, Impey RW, Klein ML (1983) J Chem Phys 79:926

26. Maier JA, Martinez C, Kasavajhala K, Wickstrom L, Hauser KE, Simmerling C (2015) J Chem Theory Comput 11:3696

27. Knowles PJ, Werner HJ (1985) Chem Phys Lett 115:259

28. Plasser F, Mewes SA, Dreuw A, González L (2017) J Chem Theory Comput 13:5343

29. Plasser F, Wormit M, Dreuw A (2014) J Chem Phys 141:024106

30. Nogueira JJ, Plasser F, González L (2017) Chem Sci 8:5682

31. Scholes GD, Ghiggino KP (1994) J Phys Chem 98:4580

32. Mai S, Marquetand P, González L (2015) Int J Quantum Chem 115:1215
33. Rodriguez-Serrano A, Daza MC, Doerr M, Marian CM (2012) Photochem Photobiol Sci 11:397

34. Weingart O (2008) Chem Phys 349:348

35. Boggio-Pasqua M, Burmeister CF, Robb MA, Groenhof G (2012) Phys Chem Chem Phys 14:7912

36. Salomon-Ferrer R, Götz AW, Poole D, Le Grand S, Walker RC (2013) J Chem Theory Comput 9:3878

37. Case DA, Berryman JT, Betz RM, Cerutti DS, Cheatham TE III, Darden TA, Duke RE, Giese TJ, Gohlke H, Goetz AW, Homeyer N, Izadi S, Janowski P, Kaus J, Kovalenko A, Lee TS, LeGrand S, Li P, Luchko T, Luo R, Madej B, Merz KM, Monard G, Needham P, Nguyen H, Nguyen HT, Omelyan I, Onufriev A, Roe DR, Roitberg A, Salomon-Ferrer R, Simmerling CL, Smith W, Swails J, Walker RC, Wang J, Wolf RM, Wu X, York DM, Kollman PA (2015) AMBER 2015. University of California, San Francisco

38. Becke AD (1993) J Chem Phys 98:5648

39. Grimme S, Antony J, Ehrlich S, Krieg H (2010) J Chem Phys 132:154104

40. Hariharan PC, Pople JA (1973) Theoret Chimica Acta 28:213

41. Francl MM, Petro WJ, Hehre WJ, Binkley JS, Gordon MS, DeFrees DJ, Pople JA (1982) J Chem Phys 77:3654

42. Ufimtsev IS, Martinez TJ (2009) J Chem Theory Comput 5:2619

43. TeraChem v 1.9, PetaChem, LLC $(2009,2015)$

44. Dunning TH Jr (1989) J Chem Phys 90:1007

45. Woon DE, Dunning TH Jr (1993) J Chem Phys 98:1358

46. Zobel JP, Nogueira JJ, González L (2017) Chem Sci 8:1482

47. Aquilante F, Autschbach J, Carlson RK, Chibotaru LF, Delcey MG, De Vico L, Galván IF, Ferré N, Frutos LM, Gagliardi L, Garavelli M, Giussani A, Hoyer CE, Li Manni G, Lischka H, Ma D, Malmqvist PÃ, Müller T, Nenov A, Olivucci M, Pedersen TB, Peng D, Plasser F, Pritchard B, Reiher M, Rivalta I, Schapiro I, Segarra-Martí J, Stenrup M, Truhlar DG, Ungur L, Valentini A, Vancoillie S, Veryazov V, Vysotskiy VP, Weingart O, Zapata F, Lindh R (2016) J Comput Chem 37:506

48. Wigner E (1932) Phys Rev 40:749

49. Møller C, Plesset MS (1934) Phys Rev 46:618

50. Werner HJ, Knowles PJ, Lindh R, Manby FR, Schätz M, Celani P, Korona T, Mitrushenkov A, Rauhut G, Adler TB, Amos RD, Bernhardsson A, Berning A, Cooper DL, Deegan MJO, Dobbyn AJ, Eckert F, Goll E, Hampel C, Hetzer G, Hrenar T, Knizia G, Käppl C, Liu Y, Lloyd AW, Mata RA, May AJ, McNicholas SJ, Meyer W, Mura ME, Nicklass A, Palmieri P, Pfläger K, Pitzer R, Reiher M, Schumann U, Stoll H, Stone AJ, Tarroni R, Thorsteinsson T, Wang M, Wolf A. MOLPRO, Version 2010.1, a package of ab initio programs

51. Richter M, Marquetand P, González-Vázquez J, Sola I, González L (2011) J Chem Theory Comput 7:1253

52. Tully JC (1990) J Chem Phys 93:1061

53. Granucci G, Persico M (2007) J Chem Phys 126:134114

54. Plasser F, Ruckenbauer M, Mai S, Oppel M, Marquetand P, González L (2016) J Chem Theory Comput 12:1207

55. Granucci G, Persico M, Zoccante A (2010) J Chem Phys 133:134111 